\title{
Edukationsbedarfe von Kindern mit Brandverletzungen und deren Eltern - Ein Scoping Review
}

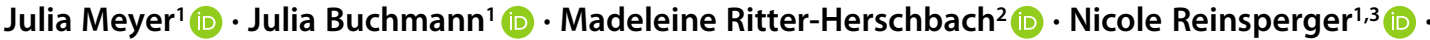 \\ Max Zilezinski ${ }^{1,2,4}$ iD
}

Eingegangen: 27. Mai 2021 / Angenommen: 16. Oktober 2021 / Online publiziert: 24. November 2021

(c) Der/die Autor(en) 2021

\section{Zusammenfassung}

Hintergrund Brandverletzungen bei Kindern sind für die ganze Familie ein traumatisches Erlebnis und mit emotionalen und psychischen Auswirkungen sowie schmerzhaften medizinischen Eingriffen verbunden. Bei Kindern mit einer schwerwiegenden thermischen Verletzung beginnt die edukative Begleitung der Familien bereits bei der Aufnahme ins Krankenhaus und setzt das Wissen um die Bedarfe der Betroffenen voraus.

Ziel Aufzeigen der Edukationsbedarfe von Kindern mit Brandverletzung und deren Eltern im stationären Setting.

Methode Es wurde ein Scoping Review durchgeführt. Die Methodik orientiert sich an dem sechsstufigen Framework von Arksey und O'Malley. Eingeschlossen wurden Studien, die Kinder mit Brandverletzungen im Alter von 0 bis 18 Jahren sowie deren Eltern während des Krankenhausaufenthaltes berücksichtigen. Die Literaturrecherche wurde in MEDLINE via PubMed durchgeführt, und Referenzen wurden gesichtet.

Ergebnisse Insgesamt konnten 3576 Studien identifiziert werden, von denen 21 Studien eingeschlossen wurden. Es ergaben sich 10 qualitative, 7 quantitative Studien sowie 4 Übersichtsarbeiten. In den meisten Studien berichten die betroffenen Kinder und deren Eltern von einem Informations- und Aufklärungsbedarf und dem Wunsch nach emotionaler und psychosozialer Entlastung. Der überwiegende Teil der Studien fokussiert die Erfahrungen von Eltern von Kindern mit Brandverletzungen.

Schlussfolgerung Für Betroffene ergeben sich im Rahmen der stationären Versorgung unterschiedliche Bedarfe zu verschiedenen Zeitpunkten. Besondere Etappen (z.B. die Entlassung, Verbandwechsel) erfordern einen erhöhten Bedarf an emotionaler und psychosozialer Entlastung, Unterstützung und/oder Information und Aufklärung.

Schlüsselwörter Scoping Review · Verbrennungen · Bedarfe $\cdot$ Krankenhausbehandlung $\cdot$ Edukation

Die Autorinnen J. Meyer und J. Buchmann teilen sich die Erstautorenschaft.

Die Autorin N. Reinsperger und der Autor M. Zilezinski teilen sich die Letztautorenschaft.

Verfügbarkeit von Daten und Materialien Alle, während dieser Recherche analysierten Studien und Materialien, sind in diesem veröffentlichten Artikel und seinen ergänzenden Anhängen enthalten.

\section{Max Zilezinski}

max.zilezinski@uk-halle.de

1 Medizinische Fakultät, Institut für Gesundheits- und Pflegewissenschaft - Masterprogramm Gesundheitsund Pflegewissenschaft, Martin-Luther-Universität Halle-Wittenberg, Magdeburger Straße 8, 06112 Halle (Saale), Deutschland
2 Universitätsmedizin Halle (Saale), AG Versorgungsforschung I Pflege im Krankenhaus, Department für Innere Medizin, Medizinische Fakultät, Martin-Luther-Universität Halle-Wittenberg, Ernst-Grube-Str. 40, 06120 Halle (Saale), Deutschland

3 Department für operative und konservative Kinderund Jugendmedizin, Universitätsmedizin Halle (Saale), Ernst-Grube-Str. 40, 06120 Halle (Saale), Deutschland

4 Medizinische Fakultät, Dorothea Erxleben Lernzentrum Halle (DELH), Projekt FORMAT, Martin-Luther-Universität Halle-Wittenberg, Magdeburger Straße 12, 06112 Halle (Saale), Deutschland 


\section{Educational needs of children with burns and their parents-A scoping review}

\section{Abstract}

Background Burn injuries of children are a traumatic experience for the whole family and are associated with emotional and psychological effects as well as painful medical interventions. Adequate support especially for children with severe burns and their parents is an important element in this process, which requires knowledge of the needs of those affected. Objective To identify the educational needs of children with burns and their parents during inpatient care.

Methods A scoping review was conducted. The methodology was guided by Arksey and O'Malley's 6-step framework. Studies that considered experiences of burn-injured children aged 0-18 years and their parents during hospitalization were included. The literature search was carried out in MEDLINE via PubMed and reference lists.

Results A total of 3576 studies were identified and 21 studies were included. 10 qualitative, 7 quantitative studies and 4 reviews resulted. In most studies, affected children and their parents reported a need for information, education and a desire for emotional and psychosocial relief. Most of the studies focused on the experiences of parents of children with burns.

Conclusion Children with burns and their parents have different needs in the context of inpatient stay. Special stages (e.g. discharge, dressing changes) require an increased need for emotional and psychosocial relief, support as well as information and education.

Keywords Scoping review $\cdot$ Burns $\cdot$ Needs $\cdot$ Acute care $\cdot$ Education

\section{Hintergrund und Problemstellung}

Die Deutsche Gesellschaft für Verbrennungsmedizin (DGV) dokumentierte im Jahr 2020, dass $73 \%$ aller Brandverletzungen bei Kindern und Jugendlichen (im Folgenden: Kinder) auftraten. Vor allem Kinder im Kleinkindalter (bis 3 Jahre) und Jugendliche sind besonders betroffen (Deutsche Gesellschaft für Verbrennungsmedizin e.V. (DGV) 2021).

Eine Verbrennung wird definiert als eine „thermische Verletzungen durch Temperaturen, welche die Regulationsfähigkeit der Haut überfordern und zu Gewebeschädigungen führen" (Deutsche Gesellschaft für Kinderchirurgie 2015, S. 5). Solche Schädigungen werden durch heiße Flüssigkeiten (Verbrühung), Dämpfe oder Gase, heiße Stoffe oder Kontaktflächen, Flammeneinwirkung und Explosionen, starke Sonneneinstrahlung, elektrischen Strom, Reibung oder chemische Noxen verursacht. Thermische Verletzungen werden nach ihrer Ausdehnung und ihrer Tiefe klassifiziert. Der Prozentsatz (\%) der verbrannten Körperoberfläche (VKOF) stellt die Ausdehnung der Verletzung dar, und mit 4 Graden kann die Tiefenausdehnung eingeschätzt werden (Deutsche Gesellschaft für Kinderchirurgie 2015).

Für Kinder sowie deren Eltern bzw. Bezugspersonen (im Folgenden: Eltern) stellt eine Brandverletzung ein traumatisches Erlebnis dar (McGarry et al. 2015; Egberts et al. 2019), das nicht selten eine multiprofessionelle Versorgung in einem Brandverletztenzentrum erfordert (Deutsche Gesellschaft für Kinderchirurgie 2015). Je nach Schweregrad der Verbrennung dauert es von der Erstversorgung im Krankenahaus bis zur vollständigen Abheilung unterschiedlich lang. Die betroffenen Kinder müssen starke Schmerzen und medizinische und pflegerische Eingriffe (z.B. Operationen, Verbandwechsel etc.) aushalten sowie einen Umgang mit den Verletzungen erlernen (Delgado Pardo et al. 2008). Eltern, die den Unfall beobachtet und/oder evtl. verursacht haben, sind durch starke Emotionen wie Angst, Traurigkeit, Hilflosigkeit sowie Scham und Schuldgefühle belastet und müssen gleichzeitig eine emotionale Unterstützung für ihr Kind sein (Delgado Pardo et al. 2008; McGarry et al. 2015; Kornhaber et al. 2018). Psychische Auswirkungen sind sowohl bei Eltern als auch bei Kindern bekannt. Demnach kann eine Brandverletzung eine Bandbreite unterschiedlicher Reaktionen hervorrufen. Dazu zählen Angstzustände, Depressionen und die posttraumatische Belastungsstörung (Delgado Pardo et al. 2008; Phillips und Rumsey 2008; McGarry et al. 2014; Egberts et al. 2018). Kinder mit einem erhöhten Anteil an Verletzungen der Körperoberfläche, der Hände und des Gesichtes haben im Nachhinein sogar eine verringerte Lebensqualität (Spronk et al. 2018). Schwerwiegende thermische Verletzungen stellen die Kinder vor enorme physiologische und psychologische Herausforderungen. Der medizinische Fortschritt und die Weiterentwicklung von Behandlungsmöglichkeiten haben dazu geführt, dass die Mortalität von Brandverletzungen deutlich gesunken ist (Kraft et al. 2012). Daraus ergibt sich jedoch meistens bei schwerwiegenden Verbrennungstraumata eine langwierige Behandlung, die den Charakter einer chronischen Erkrankung einnimmt. Mit der Schwere der Verbrennung wachsen auch das Ausmaß der Behandlung und die daraus resultierenden Edukationsbedarfe. Es gibt zwar etablierte Initiativen (z.B. Paulinchen e. V.), welche Familien mit brandverletzten Kindern beraten und begleiten, jedoch setzen diese 
Edukationsangebote erst im poststationären Setting an (Paulinchen e. V. 2021). Die Edukation und die Spättherapie v.a. bei schwerwiegenden thermischen Verletzungen beginnen jedoch bereits mit der Aufnahme ins Krankenhaus.

Unter Edukation werden vielfältige, gezielte psychologische und pädagogische Maßnahmen verstanden, welche die Selbstpflegefähigkeit und Selbstpflegekompetenz der Patientinnen und Patienten stärken (Schewior-Popp et al. 2017). Der in diesem Scoping Review verwendete Begriff bezieht sich v. a. auf die verschiedenen Funktionen, die Edukation einnehmen kann (Zegelin et al. 2009; Schaeffer 2012). Für die Edukationsbedarfe wurden folgende Kategorien aus der Literatur durch das Studienteam extrahiert:

- informieren,

- fördern,

- Hilfe planen,

- unterstützen,

- rückmelden,

- vernetzen und vermitteln,

- entlasten.

Für eine adäquate Versorgung setzt es ein Verständnis vom Fachpersonal für die Bedarfe an geeigneten Unterstützungsmaßnahmen von Betroffenen im akutstationären Setting voraus.

\section{Forschungsfrage}

Vor dem Hintergrund der Problemstellung ergibt sich daraus folgende Fragestellung:

Welche Edukationsbedarfe bestehen für Kinder mit Brandverletzungen und deren Eltern in der stationären Behandlung?

\section{Methode}

Ein Scoping Review eignet sich besonders gut, um Themen zu einem bestimmten Forschungsfeld breit und divers abzubilden. Das übergeordnete Ziel des Scoping Review besteht darin, in der Zusammenarbeit mit den Betroffenen und dem Fachpersonal in einem akutstationären Setting ein Edukationsangebot zu entwickeln. Aus diesem Grund kann der Scoping Review als ein erster Schritt zur Implementierung einer komplexen Intervention nach dem Medical Research Council (MRC) Framework verstanden werden (Shahsavari et al. 2020). Er gibt einen ersten Überblick über das vorhandene Forschungsfeld und die adressierten Edukationsbedarfe, ohne die Berücksichtigung methodischer Qualität der Studien.
Der Scoping Review orientiert sich an der methodischen Anleitung nach Arksey und O'Malley (2005), welche in 6 Schritte gegliedert wird: (1) Identifizierung der Forschungsfrage(n); (2) Identifizierung relevanter Studien; (3) Studienauswahl; (4) Darstellung der Daten; (5) Zusammenstellung, Zusammenfassung und Berichterstattung der Ergebnisse; (6) Beratung (optional).

\section{Suchstrategie}

Für die Beantwortung der Fragestellung wurde im Rahmen eine Suche in der Datenbank MEDLINE via PubMed im Juli und August 2020 durchgeführt (J.M. und M.Z.). Zusätzlich wurden die Referenzlisten der durch die Suche identifizierten Studien nach weiteren Referenzen sowie Fachjournale (z. B. Burns) durchsucht (J.M.). Graue Literatur ist nicht berücksichtig worden. Dem folgenden Absatz kann die Syntax der Suchstrategie entnommen werden.

Syntax der Suchstrategie MEDLINE via PubMed ((information need*)) OR (psychoeducation need*)) OR (education need*)) OR (parents need*)) OR (information prefer*)) OR (psychoeducation prefer*)) OR (education prefer*)) OR (parents prefer*)) OR (information request)) OR (psychoeducation request)) OR (education request)) OR (parents request)) OR (information wish)) OR (psychoeducation wish)) OR (education wish)) OR (parents wish)) OR (information demand)) OR (psychoeducation demand)) OR (education demand)) OR (information expect*)) OR (psychoeducation expect)) OR (education expect)) OR (decision need)) OR (decision support needs)) OR (decision making need)) OR (decision-making need)) OR (decision prefer)) OR (decision support prefer)) OR (parents perspective)) OR (parents concerns)) OR (parents attitude)) OR (parents opinion)) OR (parents interest)) OR (need assessment)) OR (treat prefer)) AND ((pediatric acute burn) OR (pediatric burns)) OR (pediatric burn injury)) OR (pediatric burn injuries)) OR (burn-injured children)) OR (pediatric burn patients)) OR (children with scalds)) OR (burn injury)) OR (burn injuries)) OR (childhood burns)) OR (patients with burns)) OR (burned children)) OR (pediatric burn care)) OR (burns[MeSH Terms])))

\section{Ein- und Ausschlusskriterien}

Die Ein- und Ausschlusskriterien der Literaturrecherche können der Tab. 1 entnommen werden. Es wurde keine Eingrenzung des Studiendesigns vorgenommen.

Um die Edukationsbedarfe von Kindern mit Brandverletzungen und deren Eltern zu identifizieren, wurden Studien mit einem hermeneutisch-interpretativen Studiendesign bevorzugt, welche die Erfahrungen, Wünsche, Bedarfe in der Versorgung während des Krankenhausaufenthaltes darstel- 
Tab. 1 Ein- und Ausschlusskriterien Suchstrategie MEDLINE via PubMed

\begin{tabular}{ll}
\hline Einschlusskriterien & Ausschlusskriterien \\
\hline Englische und deutsche Publikationen & Prävention von thermischen Verletzungen (v. a. Sonnenbrand) \\
Population: Kinder im Alter von 0 bis 18 Jahre & Erste-Hilfe-Maßnahmen bei thermischen Verletzungen \\
Eltern und/oder Sorgeberechtigte mit einem brandverletzten Kind & Setting außerhalb des Krankenhauses \\
Outcomes: Erfahrungen, Wünsche, Bedarfe in der Versorgung wäh- & \\
rend des Krankenhausaufenthaltes & \\
Publikationsjahr: 2000 und später & \\
\hline
\end{tabular}

len. Da vor allem die Bedarfe im stationären Setting von Interesse waren, wurden Studien, welche die Prävention von Brandverletzungen und Erste-Hilfe Maßnahmen adressieren, sowie Studien, die sich alleinig auf das außerklinische Setting beziehen, ausgeschlossen.

\section{Datenextraktion und Synthese}

Die Merkmale der Studien wurden von einer Autorin (J.M.) in einer Datentabelle zusammengefasst, analysiert und zur Qualitätssicherung von M.Z. überprüft. Die Inhalte der final eingeschlossenen Artikel wurden anhand der 7 Funktionen der Edukation thematisch zusammengefasst (Schaeffer 2012).

\section{Ergebnisse}

\section{Recherche und Studienauswahl}

Die Literaturrecherche in MEDLINE via PubMed wurde unabhängig durchgeführt (J.M. und M.Z.). Die Auswahl der Studien wurde durch die Sichtung des Titels, des Abstract und des Volltextes vorgenommen. Jegliche Widersprüchlichkeiten im Auswahlprozess, die zwischen der Autorin und dem Autor entstanden sind, wurden durch eine Konsensfindung in einer gemeinsamen Diskussion gelöst.

Die Recherche in MEDLINE via PubMed ergab insgesamt 3560 Treffer. Zusätzlich wurden 16 Studien aus relevanten Fachjournalen oder Referenzlisten identifiziert $(n=3576)$. Eine doppelte Veröffentlichung ist exkludiert worden. Nach einem Titel- und Abstract-Screening wurden 3482 Titel ausgeschlossen. Daraus ergaben sich 93 potenziell relevante Volltexte. Nach Prüfung der Volltexte auf Eignung wurden schließlich 21 Publikationen eingeschlossen (Abb. 1).

\section{Merkmale der eingeschlossenen Studien}

Die Studiencharakteristika mit ihren wesentlichen Merkmalen sind in Tab. 2. dargestellt. Die 21 Publikationen wurden in folgenden Ländern durchgeführt: Australien (McGarry et al. 2014, 2015), Neuseeland (Smith et al. 2011), Ma- rokko (El Hamaoui et al. 2006), Indien (Ravindran et al. 2013), Großbritannien (Phillips und Rumsey 2008; Morley et al. 2017; Andrews et al. 2018; Kornhaber et al. 2018; Coy et al. 2019), Niederlande (Bakker et al. 2013; Egberts et al. 2018, 2019; Suurmond et al. 2020), Spanien (Delgado Pardo et al. 2008), Brasilien (Pan et al. 2018), USA (Smith et al. 2004; Stoddard et al. 2017), Schweden (Willebrand und Sveen 2016; Willebrand et al. 2018) und Norwegen (Lernevall et al. 2020). Alle Publikationen waren in englischer Sprache verfasst. Es wurden 10 qualitative (Smith et al. 2004; Ravindran et al. 2013; McGarry et al. 2014, 2015; Morley et al. 2017; Andrews et al. 2018; Coy et al. 2019; Egberts et al. 2018, 2019; Suurmond et al. 2020), 7 quantitative Studien (El Hamaoui et al. 2006; Delgado Pardo et al. 2008; Phillips und Rumsey 2008; Smith et al. 2011; Willebrand und Sveen 2016; Stoddard et al. 2017; Willebrand et al. 2018) und 4 Übersichtsarbeiten eingeschlossen (Bakker et al. 2013; Kornhaber et al. 2018; Pan et al. 2018; Lernevall et al. 2020). Die qualitativen Studien fokussierten sich hauptsächlich auf die Erfahrungen von Eltern, Kindern und/oder Pflegefachpersonen. Die quantitativen Studien untersuchten mit diagnostischen Verfahren und Assessmentinstrumenten v. a. die emotionalen und psychischen Auswirkungen auf Kinder mit Brandverletzungen und deren Eltern (El Hamaoui et al. 2006; Delgado Pardo et al. 2008; Phillips und Rumsey 2008; Stoddard et al. 2017; Willebrand et al. 2018). Von den 4 Übersichtsarbeiten (Bakker et al. 2013; Kornhaber et al. 2018; Pan et al. 2018; Lernevall et al. 2020) inkludierten 3 Übersichtsarbeiten unterschiedliche Studiendesigns (Bakker et al. 2013; Pan et al. 2018; Lernevall et al. 2020) und eine ausschließlich qualitative Studie (Kornhaber et al. 2018).

\section{Merkmale der Studienpopulation}

Von den qualitativen Studien adressierten zwei Studien die Kinder (McGarry et al. 2014; Egberts et al. 2018), fünf die Eltern (Ravindran et al. 2013; McGarry et al. 2015; Morley et al. 2017; Andrews et al. 2018; Egberts et al. 2019), eine Studie die Pflegefachpersonen (Suurmond et al. 2012), und drei Studien hatten eine gemischte Studienpopulation (Smith et al. 2004; Kornhaber et al. 2018; Coy et al. 2019). Eine Übersichtsarbeit schloss die Eltern (Lernevall 
Abb. 1 Flowchart: MEDLINERecherche

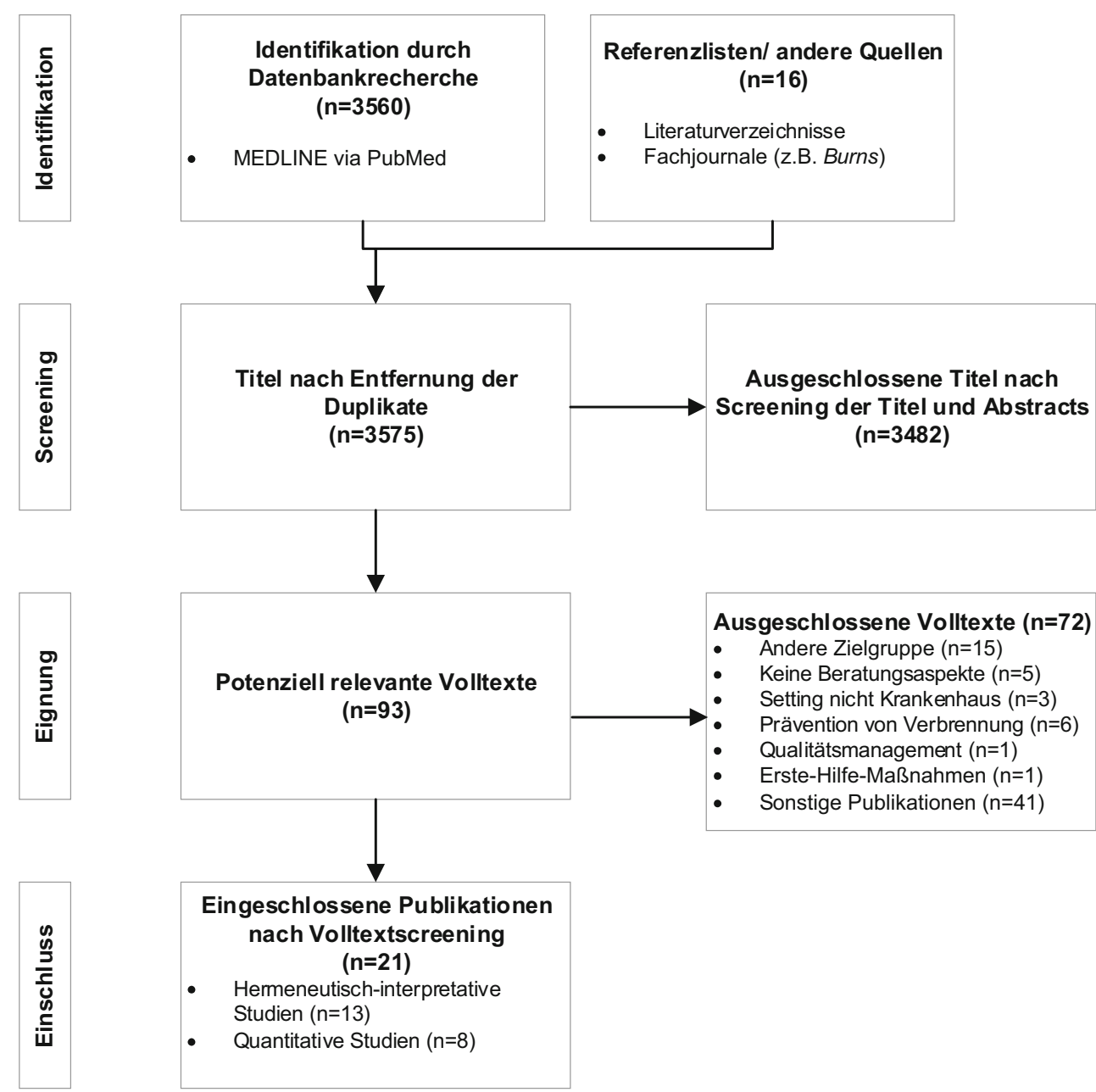

et al. 2020) und zwei Übersichtsarbeiten schlossen sowohl Kinder als auch Eltern ein (Bakker et al. 2013; Pan et al. 2018). Die meisten quantitativen Studien wurden mit Eltern durchgeführt (El Hamaoui et al. 2006; Phillips und Rumsey 2008; Willebrand und Sveen 2016; Willebrand et al. 2018), zwei mit Kindern (Delgado Pardo et al. 2008; Stoddard et al. 2017) und eine mit einer gemischten Studienpopulation (Smith et al. 2011). In den Studien reichte die Altersspanne der Kinder von 0 bis 18 Jahren.

\section{Identifizierte Themen im Rahmen der stationären Versorgung}

Aus den 21 Studien lassen sich unterschiedliche Bedarfe und Wünsche zur Unterstützung und Edukation von Kindern mit Brandverletzungen und deren Eltern skizzieren. Die Abb. 2 zeigt eine inhaltliche Zusammenfassung der Themen im zeitlichen Verlauf der Behandlung. Es zeigt sich, dass in den Studien am häufigsten die Funktionen,,entlasten“ und ,unterstützen“ thematisiert werden. Die Funktion ,rückmelden“ wurde selten benannt. Des Weiteren wur- den mit Ausnahme einer Studie, in allen Studien mindestens zwei und mehr Funktionen beschreiben.

\section{Informations- und Aufklärungsbedarf}

Der Informations- und Aufklärungsbedarf wurde in sieben Studien zusammengefasst und gilt für Betroffene als zentrales Element der Versorgung (Smith et al. 2004, 2011; McGarry et al. 2015; Morley et al. 2017; Willebrand et al. 2018; Coy et al. 2019; Lernevall et al. 2020). In drei Studien wurde von den Eltern berichtet, dass die Informationsweitergabe sensibel und zum richtigen Zeitpunkt erfolgen sowie vollständig sein sollte (McGarry et al. 2015; Coy et al. 2019; Lernevall et al. 2020). In sechs Studien gaben die Kinder und Eltern an, dass sie sich Information und Aufklärung zu folgenden Themen wünschen: Stationsroutine/ Erwartungen, physische und psychische Beeinträchtigung des Kindes, Wundmanagement, Fortschritt/Heilung, Bewegung, emotionale Verarbeitung und Kommunikationsstrategien und Entlassung (Smith et al. 2004, 2011; McGarry 
Tab. 2 Studiencharakteristika und Kurzdarstellung der Hauptergebnisse

\begin{tabular}{|c|c|c|c|c|c|}
\hline $\begin{array}{l}\text { Autorinnen } \\
\text { und Autoren } \\
\text { (Jahr), Land }\end{array}$ & $\begin{array}{l}\text { Forschungs- } \\
\text { design }\end{array}$ & $\begin{array}{l}\text { Studiengröße } \\
\text { und -po- } \\
\text { pulation }\end{array}$ & Studienziel(e) & Ergebnisse & $\begin{array}{l}\text { Einteilung } \\
\text { nach Funktion } \\
\text { nach Schaeffer } \\
\text { (2012) }\end{array}$ \\
\hline $\begin{array}{l}\text { Smith et al. } \\
(2011) \text {, } \\
\text { Neuseeland }\end{array}$ & Quantitativ & $\begin{array}{l}n=30 \\
\text { Eltern der } \\
\text { Kinder } \\
(0-15 \mathrm{~J} .) \\
\text { und Pfle- } \\
\text { gefachper- } \\
\text { sonen }\end{array}$ & $\begin{array}{l}\text { Vergleich zwischen der } \\
\text { Auffassung von Eltern und } \\
\text { Pflegefachpersonen über die } \\
\text { Sinnhaftigkeit von } \\
\text { Interventionen rund um den } \\
\text { Verbandwechsel (VW) }\end{array}$ & $\begin{array}{l}\text { Bedarfe } \\
\text { Perspektive der Eltern } \\
\text { Informationsweitergabe vor dem VW } \\
\text { Kommunikation zwischen allen Beteilig- } \\
\text { ten } \\
\text { Emotionale Vorbereitung auf den VW } \\
\text { Partizipation } \\
\text { Perspektive der Pflegefachpersonen } \\
\text { Mehr Sedativa } \\
\text { Weniger Wartezeiten } \\
\text { Unterstützung beim VW } \\
\text { Dokumentation von hilfreichen Coping- } \\
\text { Strategien }\end{array}$ & $\begin{array}{l}\text { Informieren } \\
\text { Fördern } \\
\text { Rückmelden } \\
\text { Entlasten }\end{array}$ \\
\hline $\begin{array}{l}\text { El Hamaoui } \\
\text { et al. (2006), } \\
\text { Marokko }\end{array}$ & Quantitativ & $\begin{array}{l}n=18 \\
\text { Mütter der } \\
\text { Kinder } \\
(4-14 \mathrm{~J} .)\end{array}$ & $\begin{array}{l}\text { Erfassung der Prävalenz von } \\
\text { Depressionen bei Müttern mit } \\
\text { einem brandverletzten Kind }\end{array}$ & $\begin{array}{l}\text { 71,4\% empfinden Schulgefühle } \\
35,7 \% \text { erfüllen die Kriterien für eine } \\
\text { schwere Depression } \\
\text { Schwere der Brandverletzung, geringer } \\
\text { sozioökonomischer Status und Komplika- } \\
\text { tionen wie Amputation oder sekundären } \\
\text { Infektionen sind mit Depression assozi- } \\
\text { iert } \\
\text { Empfehlung einer Einzel- oder Gruppen- } \\
\text { therapie (während/nach Krankenhausauf- } \\
\text { enthalt) }\end{array}$ & $\begin{array}{l}\text { Vernetzen und } \\
\text { vermitteln } \\
\text { Entlasten }\end{array}$ \\
\hline $\begin{array}{l}\text { Ravindran } \\
\text { et al. (2013), } \\
\text { Indien }\end{array}$ & Qualitativ & $\begin{array}{l}n=22 \\
\text { Familienmit- } \\
\text { glieder der } \\
\text { Kinder } \\
\text { (8M.-9J.) }\end{array}$ & $\begin{array}{l}\text { Beschreibung der Elternrolle } \\
\text { von Eltern mit einem } \\
\text { brandverletzten Kind }\end{array}$ & $\begin{array}{l}\text { Mit dem Kind das Trauma erleben und } \\
\text { erleiden (Religion/Glaube, Schwere der } \\
\text { Verletzung realisieren, Unterstützung er- } \\
\text { halten, emotional und physisch mitleiden) } \\
\text { Das Leben des Kindes aufrechterhalten } \\
\text { (Ressourcen mobilisieren, eine große } \\
\text { Wunde versorgen, Kind als oberste Prio- } \\
\text { rität) } \\
\text { Das Kind vor einem Stigma bewahren }\end{array}$ & $\begin{array}{l}\text { Fördern } \\
\text { Unterstützen } \\
\text { Entlasten }\end{array}$ \\
\hline $\begin{array}{l}\text { Morley et al. } \\
\text { (2017), } \\
\text { Großbritan- } \\
\text { nien }\end{array}$ & Qualitativ & $\begin{array}{l}n=5 \\
\text { Mütter der } \\
\text { Kinder } \\
(<5 \mathrm{~J} .)\end{array}$ & $\begin{array}{l}\text { Erfassung der individuellen } \\
\text { Erfahrungen von Müttern } \\
\text { während des VW }\end{array}$ & $\begin{array}{l}\text { Aufgrund der Mutterrolle besteht das } \\
\text { Bedürfnis, Verantwortung übernehmen zu } \\
\text { müssen } \\
\text { Emotionale Synchronität mit dem Kind } \\
\text { (Mitleiden) } \\
\text { Bedarfe } \\
\text { Information und Erwartung mitgeteilt } \\
\text { bekommen } \\
\text { Beziehungsaufbau mit der Pflegefach- } \\
\text { kraft, die den VW durchführt }\end{array}$ & $\begin{array}{l}\text { Informieren } \\
\text { Fördern } \\
\text { Hilfe planen } \\
\text { Unterstützen } \\
\text { Rückmelden } \\
\text { Entlasten }\end{array}$ \\
\hline $\begin{array}{l}\text { Suurmond } \\
\text { et al. (2012), } \\
\text { Niederlande }\end{array}$ & Qualitativ & $\begin{array}{l}n=17 \\
\text { Pflegefach- } \\
\text { personen }\end{array}$ & $\begin{array}{l}\text { Unterstützungsmaßnahmen } \\
\text { von ethnischen Minderheiten } \\
\text { (türkische und marokkanische } \\
\text { Eltern) }\end{array}$ & $\begin{array}{l}\text { Sprachliche Barrieren } \\
\text { Erschwerte Hautbeurteilung } \\
\text { Unsicherheiten bei Pflegefachkräften }\end{array}$ & Rückmelden \\
\hline
\end{tabular}


Tab. 2 (Fortsetzung)

\begin{tabular}{|c|c|c|c|c|c|}
\hline $\begin{array}{l}\text { Autorinnen } \\
\text { und Autoren } \\
\text { (Jahr), Land }\end{array}$ & $\begin{array}{l}\text { Forschungs- } \\
\text { design }\end{array}$ & $\begin{array}{l}\text { Studiengröße } \\
\text { und -po- } \\
\text { pulation }\end{array}$ & Studienziel(e) & Ergebnisse & $\begin{array}{l}\text { Einteilung } \\
\text { nach Funktion } \\
\text { nach Schaeffer } \\
\text { (2012) }\end{array}$ \\
\hline $\begin{array}{l}\text { Delgado } \\
\text { Pardo et al. } \\
\text { (2008), } \\
\text { Spanien }\end{array}$ & Quantitativ & $\begin{array}{l}n=83 \\
\text { Kinder } \\
(1-17 \mathrm{~J} .)\end{array}$ & $\begin{array}{l}\text { Erfassung der Unfallarten der } \\
\text { Brandverletzung (BV), die } \\
\text { emotionale Reaktion, das } \\
\text { Verhalten sowie Folgeschäden } \\
\text { der Kinder }\end{array}$ & $\begin{array}{l}\text { Die meisten Unfälle passieren zu Hause } \\
\text { (kochendes Wasser, Kaffee, Öl, Feuer) } \\
\text { Angstlevel erhöht, nicht dauerhaft (,state } \\
\text { anxiety“) } \\
\text { Angst steigt mit dem Alter der Kinder } \\
\text { Reaktionen auf den Unfall: erhöhtes } \\
\text { Bindungsverhalten, starkes Heimweh, } \\
\text { vermehrtes Weinen } \\
\text { Keine erfassten Folgeschäden }\end{array}$ & $\begin{array}{l}\text { Unterstützen } \\
\text { Entlasten }\end{array}$ \\
\hline $\begin{array}{l}\text { Pan et al. } \\
(2018), \\
\text { Brasilien }\end{array}$ & Review & $n=13$ & $\begin{array}{l}\text { Synthetisierung } \\
\text { wissenschaftlicher } \\
\text { Erkenntnisse in der Literatur } \\
\text { über Schulintegration von } \\
\text { brandverletzten Kindern }\end{array}$ & $\begin{array}{l}\text { Bedarfe } \\
\text { Kommunikation zwischen den Akteuren } \\
\text { essenziell } \\
\text { (Emotionale) Vorbereitung auf die } \\
\text { Schulintegration noch während des Kran- } \\
\text { kenhausaufenthaltes } \\
\text { Unterstützung durch Eltern } \\
\text { Schulreintegrationsprogramme }\end{array}$ & $\begin{array}{l}\text { Informieren } \\
\text { Fördern } \\
\text { Hilfe planen } \\
\text { Unterstützen } \\
\text { Vernetzen und } \\
\text { vermitteln } \\
\text { Entlasten }\end{array}$ \\
\hline $\begin{array}{l}\text { Smith et al. } \\
(2004), \text { USA }\end{array}$ & Qualitativ & $\begin{array}{l}n=1 \mathrm{Ju}- \\
\text { gendlicher } \\
(14 \mathrm{~J} .) \\
n=1 \text { Pfle- } \\
\text { gefachper- } \\
\text { son }\end{array}$ & $\begin{array}{l}\text { Erfahrung und Erinnerungen } \\
\text { eines Jugendlichen mit einer } \\
\text { schweren BV und einer } \\
\text { Pflegefachkraft im } \\
\text { Versorgungsprozess }\end{array}$ & $\begin{array}{l}\text { Behandlungsteam und Familie nehmen } \\
\text { wichtige Rollen in der Unterstützung ein } \\
\text { Extreme Situation, die physische und } \\
\text { psychische Auswirkungen hat } \\
\text { Entlassung als wichtiger Behandlungs- } \\
\text { schritt }\end{array}$ & $\begin{array}{l}\text { Hilfe planen } \\
\text { Unterstützen } \\
\text { Vernetzen und } \\
\text { vermitteln } \\
\text { Entlasten }\end{array}$ \\
\hline $\begin{array}{l}\text { Willebrand } \\
\text { et al. (2018), } \\
\text { Schweden }\end{array}$ & Quantitativ & $\begin{array}{l}n=62 \\
\text { Eltern der } \\
\text { Kinder } \\
(0-15 \mathrm{~J} .)\end{array}$ & $\begin{array}{l}\text { Erfassung der Qualität der } \\
\text { Behandlung von } \\
\text { brandverletzten Kindern aus } \\
\text { Sicht der Eltern }\end{array}$ & $\begin{array}{l}\text { Eltern haben insgesamt eine hohe Quali- } \\
\text { tät der Behandlung angegeben } \\
\text { Bedarfe } \\
\text { Anbindung an das Krankenhaus nach der } \\
\text { Entlassung } \\
\text { Informationen zur Stationsroutine }\end{array}$ & $\begin{array}{l}\text { Informieren } \\
\text { Hilfe planen } \\
\text { Rückmelden }\end{array}$ \\
\hline $\begin{array}{l}\text { Willebrand } \\
\text { und Sveen } \\
\text { (2016), } \\
\text { Schweden }\end{array}$ & Quantitativ & $\begin{array}{l}n=101 \\
\text { Eltern der } \\
\text { Kinder } \\
(0-18 \mathrm{~J} .)\end{array}$ & $\begin{array}{l}\text { Erfassung, ob ein Mangel an } \\
\text { Unterstützung nach der } \\
\text { Entlassung von } \\
\text { brandverletzten Kindern und } \\
\text { deren Eltern vorhanden war, } \\
\text { und wenn ja, welche Art von } \\
\text { Mangel }\end{array}$ & $\begin{array}{l}21 \% \text { geben einen Mangel an } \\
\text { Bedarfe } \\
\text { Psychologische, medizinische, soziale, } \\
\text { familiäre Unterstützung }\end{array}$ & $\begin{array}{l}\text { Unterstützen } \\
\text { Vernetzen und } \\
\text { vermitteln } \\
\text { Entlasten }\end{array}$ \\
\hline $\begin{array}{l}\text { Coy et al. } \\
(2019) \text {, } \\
\text { Großbritan- } \\
\text { nien }\end{array}$ & Qualitativ & $\begin{array}{l}n=14 \\
\text { Eltern der } \\
\text { Kinder } \\
(14 \mathrm{M} .-5 \mathrm{~J} .) \\
n=10 \\
\text { Fachpersonal }\end{array}$ & $\begin{array}{l}\text { Erfassung der Erfahrungen } \\
\text { von Eltern und Fachpersonal } \\
\text { in der Versorgung von } \\
\text { Kindern mit BV } \\
\text { Erstellung eines Kurzfilms } \\
\text { Hilfsmaßnahmen und } \\
\text { Unterstützungsangebote } \\
\text { herausfiltern }\end{array}$ & $\begin{array}{l}\text { Leben umgestürzt/umgeworfen } \\
\text { Beginnende/hereinbrechende Realität } \\
\text { Emotionale Achterbahn fahren } \\
\text { Nachbeben } \\
\text { Anpassung an eine neue Normalität } \\
\text { Bedarfe } \\
\text { Informationen zur richtigen Zeit und } \\
\text { sensibel vermitteln } \\
\text { Angemessene Kommunikation } \\
\text { Beachtung der emotionalen Betroffenheit } \\
\text { der Eltern und Kinder } \\
\text { Informationen zum VW } \\
\text { Mehr Rückmeldung/Transparenz zum } \\
\text { Heilungsprozess } \\
\text { Emotionale Unterstützung } \\
\text { Informationsaustausch mit anderen Fami- } \\
\text { lien }\end{array}$ & $\begin{array}{l}\text { Informieren } \\
\text { Fördern } \\
\text { Hilfe planen } \\
\text { Unterstützen } \\
\text { Rückmelden } \\
\text { Vernetzen und } \\
\text { vermitteln } \\
\text { Entlasten }\end{array}$ \\
\hline
\end{tabular}


Tab. 2 (Fortsetzung)

\begin{tabular}{|c|c|c|c|c|c|}
\hline $\begin{array}{l}\text { Autorinnen } \\
\text { und Autoren } \\
\text { (Jahr), Land }\end{array}$ & $\begin{array}{l}\text { Forschungs- } \\
\text { design }\end{array}$ & $\begin{array}{l}\text { Studiengröße } \\
\text { und -po- } \\
\text { pulation }\end{array}$ & Studienziel(e) & Ergebnisse & $\begin{array}{l}\text { Einteilung } \\
\text { nach Funktion } \\
\text { nach Schaeffer } \\
\text { (2012) }\end{array}$ \\
\hline $\begin{array}{l}\text { Egberts et al. } \\
(2018) \text {, } \\
\text { Niederlande }\end{array}$ & Qualitativ & $\begin{array}{l}n=8 \\
\text { Kinder } \\
(12-17 \mathrm{~J} .)\end{array}$ & $\begin{array}{l}\text { Erfassung der Erfahrungen } \\
\text { von Kindern mit einer BV und } \\
\text { deren Folgen }\end{array}$ & $\begin{array}{l}\text { Lebhafte Erinnerungen } \\
\text { Unterstützung durch die Eltern war sehr } \\
\text { bedeutend und wichtig }\end{array}$ & $\begin{array}{l}\text { Fördern } \\
\text { Unterstützen } \\
\text { Entlasten }\end{array}$ \\
\hline
\end{tabular}

$\begin{array}{llll}\begin{array}{l}\text { Andrews } \\ \text { et al. (2018), }\end{array} & \text { Qualitativ } & n=22 & \begin{array}{l}\text { Erfassung der Erfahrungen im } \\ \text { Elternteilen }\end{array} \\ \begin{array}{ll}\text { Wroßbritan- } \\ \text { nien }\end{array} & \begin{array}{l}\text { der Kinder } \\ (1-9 \mathrm{~J} .)\end{array} & \begin{array}{l}\text { Narbenmanagement zur } \\ \text { Fortführung der Therapie im } \\ \text { häuslichen Umfeld }\end{array}\end{array}$

Psychosoziale Auswirkungen der BV

Entwicklung individueller Coping-Strategien

Entlassung

- Zurück zur Normalität kehren

- Traumatisches Erlebnis

Übernahme der therapeutischen Rolle

- Praktische Realität der Behandlung

- Krankenhaustermine wahrnehmen

- Arbeit aufteilen $\hat{=}$ Last verteilen

- Routine entwickeln

Emotionale Verarbeitung während der

Versorgung

- Umgang mit den Emotionen des Kindes

- Umgang mit eigenen Emotionen (Retraumatisierung, Anpassung vom Behandlungsregime)

- Zukunftsvorstellungen

Bakker et al. Review $\quad n=75 \quad$ Überblick der Studien zu (2013), psychischen Auswirkungen einer BV bei Kindern und

Niederlande deren Eltern

Ergebnisse aus Studien mit dem Fokus auf die Kinder

Kinder erleben Angst und traumatischen Stress

Erhöhtes Risiko für Depressionen und Stimmungsschwankungen

Kinder kämpfen mit internalisierenden und externalisierenden Problemen

Schwierigkeiten mit sozialen Kontakten und Verhaltensweisen

Selbstwertgefühl verringert

Generell keine Unterschiede in der Körperwahrnehmung, jedoch Assoziation mit Schwere der Verbrennung bzw. Narben und dem weiblichen Geschlecht

Fehlzeiten in der Schule - adäquate Schulintegration

Fehlende Evidenz zu folgenden Themen: Schlafprobleme, Entwicklungsstörungen, kognitive Einschränkungen, Sexualität

Ergebnisse aus Studien mit dem Fokus auf die Eltern

Eltern erleben Angst und traumatischen Stress

Erhöhtes Risiko für Depressionen

Familiäre Funktion ist wichtig für den Erfolg der Behandlung 
Tab. 2 (Fortsetzung)

\begin{tabular}{|c|c|c|c|c|c|}
\hline $\begin{array}{l}\text { Autorinnen } \\
\text { und Autoren } \\
\text { (Jahr), Land }\end{array}$ & $\begin{array}{l}\text { Forschungs- } \\
\text { design }\end{array}$ & $\begin{array}{l}\text { Studiengröße } \\
\text { und -po- } \\
\text { pulation }\end{array}$ & Studienziel(e) & Ergebnisse & $\begin{array}{l}\text { Einteilung } \\
\text { nach Funktion } \\
\text { nach Schaeffer } \\
(2012)\end{array}$ \\
\hline $\begin{array}{l}\text { Stoddard } \\
\text { et al. (2017), } \\
\text { USA }\end{array}$ & Quantitativ & $\begin{array}{l}n=42 \\
\text { Kinder } \\
(1-4 \mathrm{~J} .)\end{array}$ & $\begin{array}{l}\text { Erfassung posttraumatischer } \\
\text { Belastungsstörungen bei } \\
\text { Kindern mit einer } \\
\text { Verbrennung }\end{array}$ & $\begin{array}{l}\text { Geringer prozentualer Anteil der Kinder } \\
\text { erfüllt alle Kriterien in beiden Assess- } \\
\text { mentinstrumenten (10 und 3\%) } \\
\text { Hoher prozentualer Anteil von Retrauma- } \\
\text { tisierung } \\
\text { Ein Drittel aller Kinder erfüllte Teilkrite- } \\
\text { rien oder ein Symptom in jedem Cluster } \\
\text { von DICA-P }\end{array}$ & $\begin{array}{l}\text { Vernetzen und } \\
\text { vermitteln } \\
\text { Entlasten }\end{array}$ \\
\hline $\begin{array}{l}\text { Phillips und } \\
\text { Rumsey } \\
\text { (2008), } \\
\text { Großbritan- } \\
\text { nien }\end{array}$ & Quantitativ & $\begin{array}{l}n=72 \\
\text { Eltern der } \\
\text { Kinder } \\
\text { (k. A.) }\end{array}$ & $\begin{array}{l}\text { Evaluierung von Bedürfnissen } \\
\text { und psychologischen } \\
\text { Unterstützungsmaßnahmen } \\
\text { für Eltern brandverletzter } \\
\text { Kinder }\end{array}$ & $\begin{array}{l}\text { Höheres Angst- und Depressionslevel } \\
\text { während der stationären Versorgung } \\
\text { Angstlevel korreliert mit Depressionsle- } \\
\text { vel } \\
\text { Starke Psychische Auswirkungen auf die } \\
\text { Eltern }\end{array}$ & $\begin{array}{l}\text { Unterstützen } \\
\text { Entlasten }\end{array}$ \\
\hline $\begin{array}{l}\text { Egberts et al. } \\
(2019) \text {, } \\
\text { Niederlande }\end{array}$ & Qualitativ & $\begin{array}{l}n=18 \\
\text { Eltern der } \\
\text { Kinder } \\
(0-16 \mathrm{~J} .)\end{array}$ & $\begin{array}{l}\text { Erfassung der Erfahrungen, } \\
\text { Erinnerungen und Emotionen } \\
\text { der Eltern an die BV ihres } \\
\text { Kindes und den } \\
\text { Krankenhausaufenthalt }\end{array}$ & $\begin{array}{l}\text { Gefahr/Bedrohung durch die BV/Unfall } \\
\text { Mitleid/Empathie mit dem Kind } \\
\text { Emotionen: Angst, Panik, Schock, Schre- } \\
\text { cken, Hilflosigkeit, Traurigkeit, Mitge- } \\
\text { fühl, Trauer } \\
\text { Höhere Vulnerabilität des Kindes } \\
\text { Zukunftsängste } \\
\text { Verantwortung für den Unfall überneh- } \\
\text { men: Schuldgefühle und -zuweisung } \\
\text { Dankbarkeit und Hoffnung }\end{array}$ & $\begin{array}{l}\text { Unterstützen } \\
\text { Entlasten }\end{array}$ \\
\hline $\begin{array}{l}\text { McGarry } \\
\text { et al. (2014), } \\
\text { Australien }\end{array}$ & Qualitativ & $\begin{array}{l}n=12 \\
\text { Kinder } \\
(8-15 \mathrm{~J} .)\end{array}$ & $\begin{array}{l}\text { Erfassung der emotionalen } \\
\text { Erfahrungen von Kindern mit } \\
\text { einer BV }\end{array}$ & $\begin{array}{l}\text { Retraumatisierung (durch VW, Op. etc.) } \\
\text { Emotionen: (Todes-)Angst, Schock, Sor- } \\
\text { gen } \\
\text { Verhaltensveränderung } \\
\text { Narbenbildung } \\
\text { Permanente negative Erinnerung an den } \\
\text { Unfall } \\
\text { Familie und Anpassung an den Alltag } \\
\text { (Schulintegration) } \\
\text { Hilfreich: Unterstützung durch Familie }\end{array}$ & $\begin{array}{l}\text { Unterstützen } \\
\text { Entlasten }\end{array}$ \\
\hline $\begin{array}{l}\text { Lernevall } \\
\text { et al. (2020), } \\
\text { Norwegen }\end{array}$ & Review & $n=7$ & $\begin{array}{l}\text { Synthese der Literatur zu } \\
\text { Unterstützungsmöglichkeiten } \\
\text { von Eltern brandverletzter } \\
\text { Kinder im Krankenhaus } \\
\text { Erfassung der } \\
\text { wahrgenommenen } \\
\text { Unterstützung aus Sicht der } \\
\text { Eltern }\end{array}$ & $\begin{array}{l}\text { Emotionales Leid } \\
\text { Angst um das Kind und vor medizini- } \\
\text { schen/pflegerischen Behandlungen (VW, } \\
\text { Op. etc.) } \\
\text { Schuld und Vorwürfe (Eltern selbst und/ } \\
\text { oder von Familienmitgliedern/Personal) } \\
\text { Information zu erhalten ist zentral (zum } \\
\text { richtigen Zeitpunkt und sensibel) } \\
\text { Hilfreich: Unterstützung durch Familie } \\
\text { oder Fachpersonal }\end{array}$ & $\begin{array}{l}\text { Informieren } \\
\text { Unterstützen } \\
\text { Entlasten }\end{array}$ \\
\hline
\end{tabular}


Tab. 2 (Fortsetzung)

\begin{tabular}{|c|c|c|c|c|c|}
\hline $\begin{array}{l}\text { Autorinnen } \\
\text { und Autoren } \\
\text { (Jahr), Land }\end{array}$ & $\begin{array}{l}\text { Forschungs- } \\
\text { design }\end{array}$ & $\begin{array}{l}\text { Studiengröße } \\
\text { und -po- } \\
\text { pulation }\end{array}$ & Studienziel(e) & Ergebnisse & $\begin{array}{l}\text { Einteilung } \\
\text { nach Funktion } \\
\text { nach Schaeffer } \\
(2012)\end{array}$ \\
\hline $\begin{array}{l}\text { McGarry } \\
\text { et al. (2015), } \\
\text { Australien }\end{array}$ & Qualitativ & $\begin{array}{l}n=21 \\
\text { Eltern der } \\
\text { Kinder } \\
(6 \mathrm{M} .-15 \mathrm{~J} .)\end{array}$ & $\begin{array}{l}\text { Untersuchung und } \\
\text { Beschreibung der } \\
\text { Erfahrungen, die Eltern im } \\
\text { Rahmen einer BV ihres } \\
\text { Kindes gemacht haben }\end{array}$ & $\begin{array}{l}\text { Retraumatisierung (durch VW, Op. etc.) } \\
\text { Erlösung durch das Fachpersonal } \\
\text { Information zu erhalten ist zentral (zum } \\
\text { richtigen Zeitpunkt und sensibel) } \\
\text { Schuld und Schamgefühle } \\
\text { Hilfreich: psychosoziale Unterstützung } \\
\text { Partnerschaftskonflikte } \\
\text { Traumatische Erfahrungen bei Op.-Vor- } \\
\text { bereitungen und VW } \\
\text { Zurück nach Hause } \\
\text { Entlassung braucht Vorbereitung (Infor- } \\
\text { mation, psychosoziale Unterstützung, } \\
\text { Vernetzung mit anderen Hilfserbringern) } \\
\text { Verhaltensveränderung beim Kind be- } \\
\text { merkt } \\
\text { Zukunftsängste }\end{array}$ & $\begin{array}{l}\text { Informieren } \\
\text { Fördern } \\
\text { Hilfe planen } \\
\text { Unterstützen } \\
\text { Vernetzen und } \\
\text { vermitteln } \\
\text { Entlasten }\end{array}$ \\
\hline $\begin{array}{l}\text { Kornhaber } \\
\text { et al. (2018), } \\
\text { Großbritan- } \\
\text { nien }\end{array}$ & Review & $n=18$ & $\begin{array}{l}\text { Synthese der Erfahrungen } \\
\text { von Eltern/Familienmitglieder } \\
\text { brandverletzter Kinder bzgl. } \\
\text { Schuldgefühlen, Scham und } \\
\text { Vorwürfen }\end{array}$ & $\begin{array}{l}\text { Zentrale Faktoren, die das Leben der } \\
\text { Betroffenen begleiten: } \\
\text { - Schuldgefühle und Grübeln über } \\
\text { Schuldgefühle } \\
\text { - Schuldzuweisung } \\
\text { - Scham und Körperbild }\end{array}$ & $\begin{array}{l}\text { Unterstützen } \\
\text { Entlasten }\end{array}$ \\
\hline
\end{tabular}

et al. 2015; Morley et al. 2017; Willebrand et al. 2018; Coy et al. 2019).

\section{Bedarf an emotionaler und psychosozialer Entlastung und Unterstützung}

Fast alle Studien berichteten darüber, dass eine Brandverletzung enorme Auswirkungen auf die psychische und emotionale Verfassung des Kindes und dessen Eltern hat (Smith et al. 2004, 2011; El Hamaoui et al. 2006; Delgado Pardo et al. 2008; Phillips und Rumsey 2008; Bakker et al. 2013; Ravindran et al. 2013; McGarry et al. 2014, 2015; Morley et al. 2017; Stoddard et al. 2017; Andrews et al. 2018; Kornhaber et al. 2018; Pan et al. 2018; Coy et al. 2019; Egberts et al. 2018, 2019; Lernevall et al. 2020).

Eine quantitative Studie untersuchte mit Assessmentinstrumenten die emotionale Reaktion und das Verhalten des Kindes auf den Unfall sowie Folgeschäden (Delgado Pardo et al. 2008).

Bei Kindern kam es zu einem erhöhten Bindungsverhalten und Heimweh. Außerdem wurde vermehrtes Weinen beobachtet (Delgado Pardo et al. 2008).

Stoddard et al. (2017) haben mit diagnostischen Verfahren den prozentualen Anteil posttraumatischer Belastungsstörungen von Kindern erfassen können (Stoddard et al. 2017). Zwar erfüllte nur ein geringer prozentualer Anteil alle Kriterien für eine posttraumatische Belastungsstörung, jedoch zeigte sich bei über einem Drittel mindestens ein Symptom (Stoddard et al. 2017).

Die Übersichtsarbeit von Bakker et al. (2013) zeigte ebenfalls, dass viele der Kinder unter Ängsten und traumatischem Stress, Depressionen, Stimmungsschwankungen sowie internalisierenden und externalisierenden Problemen leiden (Bakker et al. 2013). Drei qualitative Studien und zwei Übersichtsarbeiten adressierten die emotionalen Erfahrungen von Kindern (Smith et al. 2004; Bakker et al. 2013; McGarry et al. 2014; Kornhaber et al. 2018; Egberts et al. 2018). Alle Studien zeigten, dass eine Brandverletzung für Kinder eine enorme emotionale Belastung darstellt, sie Angst haben und traumatischen Stress erleben.

Schmerzhafte medizinische Eingriffe (Operationen, Verbandwechsel etc.) nehmen einen Großteil der Versorgung von brandverletzten Kindern ein. Vier Studien berichteten, dass die Eingriffe immer wieder eine Retraumatisierung hervorrufen können und bei schlechter Vorbereitung, Durchführung und Nachbesprechung die emotionalen und psychischen Auswirkungen einer Brandverletzung verstärken (McGarry et al. 2014, 2015; Kornhaber et al. 2018; Coy et al. 2019).

El Hamaoui et al. (2006) haben in einer quantitativen Erhebung anhand der 4. Auflage des Diagnostic and Statistical Manual of Mental Disorders (DSM-IV) die Prävalenz von Depressionen bei Müttern mit einem brandverletzten Kind erfasst (El Hamaoui et al. 2006). 


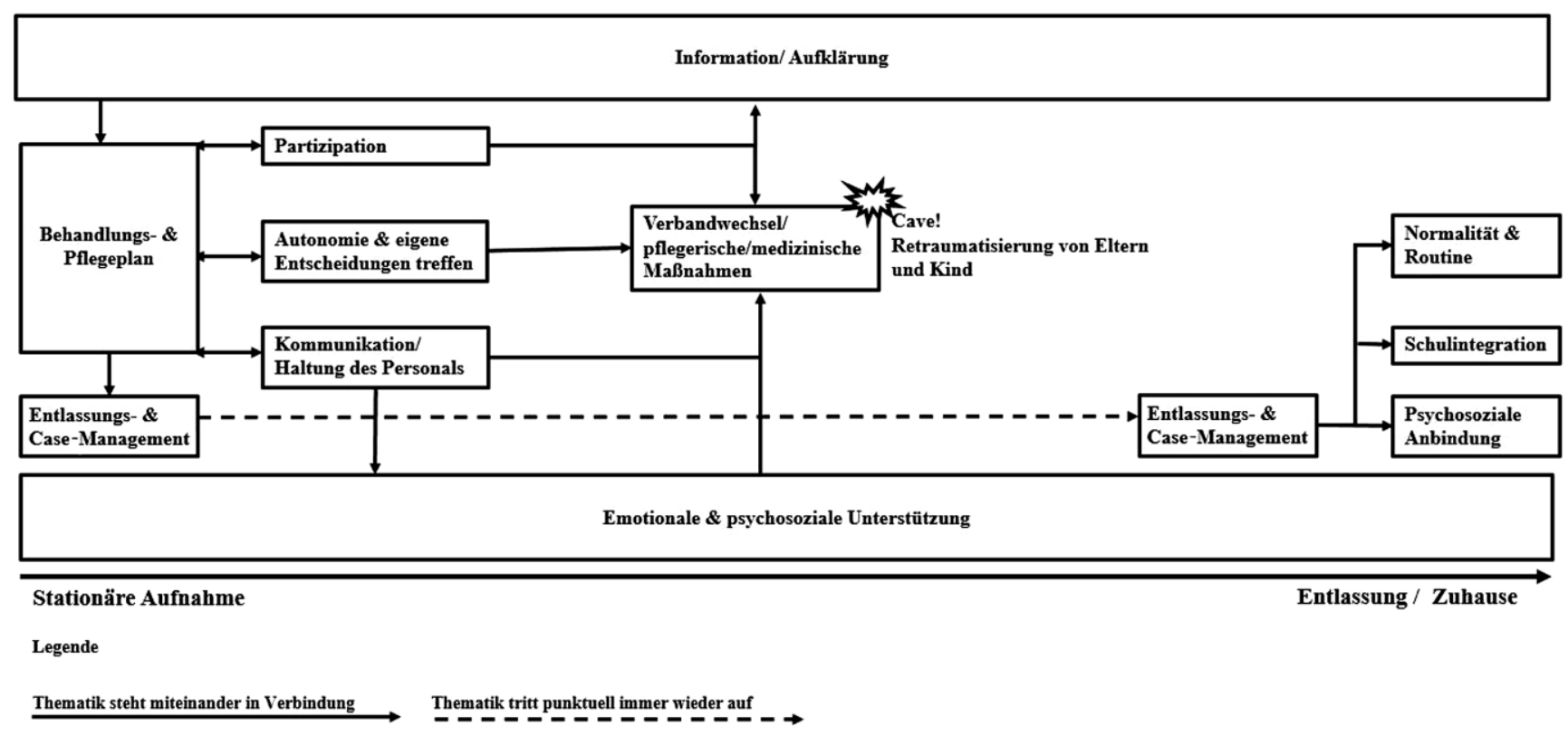

Abb. 2 Inhaltliche Zusammenfassung der Studien im zeitlichen Verlauf der Behandlung

Die emotionalen Reaktionen von Eltern auf die Brandverletzung und die Versorgung ihrer Kinder wurden v. a. in qualitativen Studien durch Interviews und Befragungen sowie in drei Übersichtsarbeiten erfasst (Bakker et al. 2013; Ravindran et al. 2013; McGarry et al. 2015; Morley et al. 2017; Andrews et al. 2018; Kornhaber et al. 2018; Coy et al. 2019; Egberts et al. 2019; Lernevall et al. 2020).

Die meisten Studien berichteten darüber, dass Eltern mit ihren Kindern emotional und psychisch mitleiden, sie Schuldgefühle und Scham entwickeln (Ravindran et al. 2013; McGarry et al. 2015; Morley et al. 2017; Kornhaber et al. 2018; Lernevall et al. 2020). In fünf Studien wird über die Angst der Eltern um ihr Kind und von traumatischem Stress berichtet (Bakker et al. 2013; McGarry et al. 2015; Andrews et al. 2018; Egberts et al. 2019; Lernevall et al. 2020).

Aus den Untersuchungen ergibt sich ein Bedarf an emotionaler Entlastung/Unterstützung durch das Fachpersonal (Pflegefachpersonen, Ärztinnen und Ärzte). Je nach Ausprägung der Auswirkungen entsteht zusätzlich ein Bedarf an psychotherapeutischer Unterstützung.

In zwei Studien gaben Eltern an, dass sie einen Mangel an emotionaler Unterstützung durch das Fachpersonal erfahren haben (Ravindran et al. 2013; Coy et al. 2019). Der Bedarf an emotionaler und psychosozialer Unterstützung der Eltern sollte zu jedem Zeitpunkt durch ein empathisches und verständnisvolles Auftreten des Personals, ohne jegliche Schuldzuweisung, gedeckt werden. Zusätzlich besteht ein Bedarf an Unterstützung und emotionaler Entlastung durch andere Familienmitglieder und Freunde (Willebrand und Sveen 2016).
In drei Studien wünschten sich Kinder und Eltern eine aufrichtige, ehrliche und empathische Haltung durch das Fachpersonal. Alle sollen Verständnis, ohne eine Schuldzuweisung, gegenüber den Eltern zeigen (Smith et al. 2004; Ravindran et al. 2013; Kornhaber et al. 2018).

\section{Bedarfe an Partizipation, Autonomie und eigene Entscheidungen treffen}

In fünf Studien wünschten sich Eltern, mehr in den Behandlungsprozess einbezogen zu werden (Smith et al. 2011; Morley et al. 2017; Willebrand et al. 2018; Coy et al. 2019; Lernevall et al. 2020). Vier Studien bezogen sich explizit auf den Verbandwechsel (Smith et al. 2011; Morley et al. 2017; Coy et al. 2019; Lernevall et al. 2020). Morley et al. (2017) stellten v.a. die Erfahrungen von Müttern in den Fokus. Die Anwesenheit bei den Verbandwechseln und die Beurteilung des Heilungsprozesses der Wunden sind für Mütter wichtig (Morley et al. 2017). Willebrand et al. (2018) merkten an, dass die Integration von Eltern in den Behandlungsprozess noch ungenügend ist und sie bessere Möglichkeiten dahingehend erhalten sollten (Willebrand et al. 2018).

Der Wunsch nach Autonomie und Partizipation wurde in einer Studie von einem brandverletzten Jugendlichen berichtet (Smith et al. 2004). Der Bedarf, eigene Entscheidungen zu treffen, lässt sich v. a. über einen direkten Kommunikationsweg zwischen den Betroffenen und dem Behandlungsteam realisieren (Smith et al. 2004). 


\section{Bedarf an Unterstützung}

Der Bedarf an Unterstützung durch das Fachpersonal, Familienmitglieder oder andere Vertrauenspersonen wurde in 7 Studien berichtet (Smith et al. 2004, 2011; Bakker et al. 2013; Ravindran et al. 2013; Willebrand et al. 2018; Egberts et al. 2018; Lernevall et al. 2020). Kinder sahen ihre Eltern und das Behandlungsteam als hilfreiche Unterstützung an (Smith et al. 2004; Egberts et al. 2018). Eltern sahen sich als Unterstützung für ihre Kinder (Smith et al. 2011), das Fachpersonal (Ravindran et al. 2013; Willebrand et al. 2018; Lernevall et al. 2020) sowie die Familie als Unterstützung für sich (Bakker et al. 2013). Pflegefachpersonen sahen Eltern als Unterstützung für die Kinder an (Smith et al. 2011).

In sieben Studien wurde die Angst vor den Reaktionen aus der Gesellschaft thematisiert sowie der Bedarf an Vorbereitung auf solche Situationen (Smith et al. 2004; Ravindran et al. 2013; McGarry et al. 2014, 2015; Andrews et al. 2018; Egberts et al. 2018, 2019).

\section{Bedarf an Rückmeldung}

Der Bedarf an Rückmeldung bzw. die Kommunikation über Fort- und Rückschritte der Wundheilung durch das Fachpersonal wurde in zwei Studien berichtet. Eltern möchten den Prozess der Heilung mitverfolgen und die Erwartungen bezüglich der Heilung transparent mitgeteilt bekommen. Eltern wünschten sich z. B. ein Fototagebuch auf der Station, strukturierte Nachbesprechungen, Sitzungen oder Gruppentherapien, die einen Austausch mit dem Behandlungsteam oder anderen Eltern ermöglichen (Smith et al. 2011; Coy et al. 2019). Kinder und Jugendlichen fanden es hilfreich, sich die Wunden anzusehen. Einerseits, um das Körpergefühl und -bild wiederherzustellen, sich daran zu gewöhnen, und andererseits, um Fortschritte zu sehen (Smith et al. 2004; Egberts et al. 2018).

\section{Entlassungs- und Case-Management sowie ambulante Versorgung}

In sieben Studien wurde über die Entlassung als Herausforderung für die Familie berichtet (Smith et al. 2004; Bakker et al. 2013; McGarry et al. 2014, 2015; Andrews et al. 2018; Pan et al. 2018; Willebrand et al. 2018). Zwei Studien adressierten das Thema Schulintegration (Bakker et al. 2013; Pan et al. 2018). Bakker et al. (2013) fanden heraus, dass Kinder mit moderaten Brandverletzungen (14\% VKOF) im Durchschnitt 38 Tage in der Schule verpassen und ungefähr zehn Tage nach Entlassung wieder in die Schule zurückgehen (Bakker et al. 2013). Pan et al. (2018) zeigten, dass diese Situation erheblichen Stress bei Eltern und Kind auslösen kann (Pan et al. 2018). Smith et al. (2004), Bakker et al. (2013), und Pan et al. (2018) vermuten, dass eine Vorbereitungsphase im stationären Setting zur erfolgreicheren Schulintegration führen kann (Smith et al. 2004; Bakker et al. 2013; Pan et al. 2018).

In vier Studien wurden die emotionalen Reaktionen der Kinder und Eltern auf die Entlassung und die damit verbundenen Bedarfe adressiert (Smith et al. 2004; McGarry et al. 2014, 2015; Andrews et al. 2018). Dabei standen Betroffene im Zwiespalt zwischen dem Wunsch nach Normalität und Routine und der Unsicherheit, die Versorgung im häuslichen Umfeld zu bewältigen (McGarry et al. 2015; Andrews et al. 2018). In dieser Phase wurde den Eltern bewusst, dass die Versorgung einen enormen Aufwand bedeutet. Waren die Eltern bis jetzt in einem geschützten Umfeld, in dem jederzeit Fachpersonal ansprechbar war, so bricht diese Komponente weg (Andrews et al. 2018). Eltern mussten dann die Aufgaben übernehmen, fühlten sich schnell überfordert und hatten das Gefühl eines Kontrollverlustes (Andrews et al. 2018). Die Angst und Sorge um das Kind spielten nunmehr die primäre Rolle, sodass Eltern ihre eigenen Bedürfnisse zurückstellten (Andrews et al. 2018).

Aus den Ergebnissen lässt sich schließen, dass sich Eltern eine Vorbereitung auf die Entlassung wünschen (McGarry et al. 2014, 2015; Andrews et al. 2018). In beiden qualitativen Studien, die explizit Kinder nach ihren Erfahrungen befragt haben, wurden keine Inhalte zur Entlassung generiert (McGarry et al. 2014; Egberts et al. 2018).

Nur Smith et al. (2004) erwähnen Inhalte, die im Rahmen des Entlassungskonzeptes angesprochen werden können. Hierzu zählen Erwartungen bezüglich Wundversorgung, Aktivitäten zu Hause, Druckkleidung, Akzeptanz in der Gemeinschaft, Schulintegration, Peer-Beziehungen, Partizipation und die emotionale Bewältigung, die, falls erforderlich, eine ambulante psychotherapeutische Behandlung für Eltern und/oder Kind beinhaltet. Eltern wünschten sich eine Anbindung an das Krankenhaus (Willebrand et al. 2018).

Andrews et al. (2018) untersuchten u.a., welche Unterstützungsmöglichkeiten sich Eltern wünschen, um die Versorgung des Kindes zu Hause aufrechtzuerhalten. Folgende Themen waren im Rahmen dieser Studie für Eltern wichtig: Informationen und Aufklärung zur Vereinbarkeit von Arbeit/Schule und Behandlung, Strategien zur Routineentwicklung/zum Einbezug des Kindes, Hilfestellungen/ Kontakte, Umgang mit den Emotionen des Kindes (Andrews et al. 2018).

Drei Studien benannten unterschiedliche Maßnahmen, die für die ambulante Versorgung brandverletzter Kinder und deren Eltern ergriffen werden können (Smith et al. 2004; El Hamaoui et al. 2006; Lernevall et al. 2020). Hierzu zählten Burn Camps für das Kind, Schulintegrationsprogramme, Selbsthilfegruppen oder Einzel- und Gruppentherapien, die durch eine Psychotherapeutin oder einen Psy- 
chotherapeuten begleitet werden (Smith et al. 2004). Lernevall et al. (2020), und El Hamaoui et al. (2006) berichteten über den Mehrwert von Gruppenangeboten für Eltern sowohl auf der Station als auch nach der Entlassung (El Hamaoui et al. 2006; Lernevall et al. 2020).

\section{Diskussion}

Zusammenfassend lässt sich sagen, dass Studien mit unterschiedlichen Designs zur Verfügung stehen. Die Mehrheit der Untersuchungen entspringt der qualitativen Forschung. Die quantitativen Studien sind v.a. deskriptive Beobachtungsstudien, die konkrete Themen (z. B. Depression, subjektive Versorgungsqualität, Verbandwechsel) in den Fokus nehmen.

Aufgrund der bleibenden und sichtbaren physischen sowie psychischen Wunden (z.B. Narbenbildung, Stigmatisierung, Isolation) ist es wichtig, das Thema Brandverletzungen bei Kindern näher zu beleuchten und vermehrt Grundlagenforschung zu betreiben, um die Versorgung der Betroffenen zu verbessern. Jugendliche scheinen stärker von emotionalen und psychischen Auswirkungen durch das Trauma betroffen zu sein (Delgado Pardo et al. 2008). Zu dieser Thematik lassen sich jedoch kaum Studien auffinden. Besonders Edukationsangebote zur Körperbildwahrnehmung, Krankheitsbewältigung und zur Schulintegration sind wichtig, um diese spezielle Zielgruppe bei ihrer Wiedereingliederung in die Gesellschaft zu unterstützen.

Bei den eingeschlossenen Studien zeigt sich, dass die Altersspanne sehr heterogen ist. Es war nicht möglich, eine Differenzierung des Edukationsbedarfes hinsichtlich des Alters vorzunehmen. Aufgrund der elterlichen Abhängigkeit von Kleinkindern und des zunehmenden Unabhängigkeitsdrangs von Jugendlichen muss jedoch davon ausgegangen werden, dass sich die Edukationsbedarfe hinsichtlich des Alters unterscheiden. Dies trifft ebenso auf den Schweregrad der Verbrennung zu, der in den Studien nicht berücksichtigt bzw. differenziert wurde. Abschließend gehen die Autorinnen und Autoren davon aus, dass sich die benannten Edukationsbedarfe in allen Altersklassen der Kinder wiederfinden und unterschiedlich gewichtet werden sowie der Schweregrad der Brandverletzung das Ausmaß des Edukationsbedarfes bestimmt.

Aufgrund des individuellen Zeit- und Ressourcenrahmens des Studienteams erfolgte die Recherche nur in einer Datenbank; graue Literatur wurde vernachlässigt. Trotz aller Sorgfalt kann somit die Vollständigkeit nicht garantiert und eine punktuelle Skizzierung nicht ausgeschlossen werden.

Da die Methodik nicht vorsieht, eine Beurteilung bzw. Bewertung der eingeschlossenen Studien vorzunehmen, können Empfehlungen für die Praxis nur limitiert graduiert werden.

\section{Schlussfolgerung und Implikationen für die Praxis}

Die Ergebnisse zeigen, dass Kinder mit einer Brandverletzung und deren Eltern herausfordernde Situationen während und nach der stationären Behandlung erleben. Im Verlauf der Behandlung einer Brandverletzung entsteht bei den betroffenen Kindern und insbesondere deren Eltern ein Grundbedürfnis nach Information und Aufklärung, welches v. a. zur Vorbereitung auf das häusliche Umfeld stärker wird und auch nach der akutstationären Behandlung bestehen bleibt.

Zur Bewältigung der Krise braucht es neben der entlastenden und fördernden Unterstützung durch die Familienmitglieder auch das Fachpersonal. Dieses ermöglicht v.a. durch Kommunikation und Information zum Wundheilungsverlauf und zur Prognose der Verletzung eine partizipative Entscheidungsfindung. Zur Deckung der Bedarfe sowie zur Förderung der Gesundheitskompetenz empfiehlt es sich, insbesondere an Brandverletztenzentren, auf den Stationen Pflegefachexpertinnen und Pflegefachexperten für Brandverletzungen einzusetzen sowie stationsunabhängige Beratungsstrukturen zu etablieren. Die strukturelle Integration von Patienteninformationszentren ist eine gute Möglichkeit, damit Eltern selbstständig Informationen einholen können und hier speziell ausgebildete Pflegefachpersonen Sprechstunden bzw. in Zusammenarbeit mit Psychologinnen und Psychologen Selbsthilfegruppen für Kinder mit Brandverletzungen anbieten.

Danksagung Wir danken den wissenschaftlichen Mitarbeitenden sowie der Leiterin des Instituts für Gesundheit- und Pflegewissenschaft der Martin-Luther-Universität Halle-Wittenberg für die spannenden Jahre im Masterstudium der Gesundheit- und Pflegewissenschaft, unter den besonderen Herausforderungen der Coronapandemie. $\mathrm{Zu}-$ sätzlich möchten wir Frau Professorin Dr. phil. Anke Steckelberg für die kompetente Methodenberatung während der Recherche im Rahmen des studentischen Forschungsprojektes danken.

Förderung Keine Förderung, Eigenmittelprojekt.

Author Contribution Literaturrecherche: J.M., M.Z., N.R. und J.B. Literaturanalyse: J.M., M.Z. Beitrag zur Konzeption und zum Design der Arbeit: J.M., M.Z., N.R., J.B. und M.R.-H. Beitrag bei der Erfassung, Analyse und der Interpretation der Literatur: J.M., M.Z. Manuskripterstellung und kritische Überarbeitung: J.M., M.Z., N.R., J.B. und M.R.-H.Genehmigung der letzten Version des Manuskripts zur Publikation: J.M., M.Z., N.R., J.B. und M.R.-H. Bereitschaft, für alle Aspekte der Arbeit Verantwortung zu übernehmen: J.M., M.Z., N.R., J.B. und M.R-H.

Funding Open Access funding enabled and organized by Projekt DEAL. 
Interessenkonflikt J. Meyer, J. Buchmann, M. Ritter-Herschbach, N. Reinsperger und M. Zilezinski geben an, dass kein Interessenkonflikt besteht.

Open Access Dieser Artikel wird unter der Creative Commons Namensnennung 4.0 International Lizenz veröffentlicht, welche die Nutzung, Vervielfältigung, Bearbeitung, Verbreitung und Wiedergabe in jeglichem Medium und Format erlaubt, sofern Sie den/die ursprünglichen Autor(en) und die Quelle ordnungsgemäß nennen, einen Link zur Creative Commons Lizenz beifügen und angeben, ob Änderungen vorgenommen wurden.

Die in diesem Artikel enthaltenen Bilder und sonstiges Drittmaterial unterliegen ebenfalls der genannten Creative Commons Lizenz, sofern sich aus der Abbildungslegende nichts anderes ergibt. Sofern das betreffende Material nicht unter der genannten Creative Commons Lizenz steht und die betreffende Handlung nicht nach gesetzlichen Vorschriften erlaubt ist, ist für die oben aufgeführten Weiterverwendungen des Materials die Einwilligung des jeweiligen Rechteinhabers einzuholen.

Weitere Details zur Lizenz entnehmen Sie bitte der Lizenzinformation auf http://creativecommons.org/licenses/by/4.0/deed.de.

\section{Literatur}

Andrews N et al (2018) Below the surface: parents' views on the factors that influence treatment adherence in paediatric burn scar management-a qualitative study. Burns 44:626-635

Arksey H, O’Malley L (2005) Scoping studies: towards a methodological framework. Int J Soc Res Methodol 8:19-32

Bakker A, Maertens KJP, Van Son MJM, Van Loey NEE (2013) Psychological consequences of pediatric burns from a child and family perspective: a review of the empirical literature. Clin Psychol Rev 33:361-371

Coy K, Brock P, Pomeroy S, Cadogan J, Beckett K (2019) A road less travelled: using experience based co-design to map children's and families' emotional journey following burn injury and identify service improvements. Burns 45:1848-1855

Delgado Pardo G, Moreno García I, Del Rosario Miralles Marrero F, Gómez Cía T (2008) Psychological impact of burns on children treated in a severe burns unit. Burns 34:986-993

Deutsche Gesellschaft für Kinderchirurgie (2015) Leitlinie „,zur Behandlung thermischer Verletzungen im Kindesalter (Verbrennung, Verbrühung)“"

Deutsche Gesellschaft für Verbrennungsmedizin (2021) Jahresbericht 2021. Für den Zeitraum Jan.-Dez. 2020

Egberts MR, Geenen R, de Jong AEE, Hofland HW, Van Loey NE (2018) The aftermath of burn injury from the child's perspective: a qualitative study. J Health Psychol 25(13-14):2464-2474

Egberts MR, Engelhard IM, de Jong AEE, Hofland HWC, Geenen R, Van Loey NEE (2019) Parents' memories and appraisals after paediatric burn injury: a qualitative study. Eur J Psychotraumatol 10(1): 1615346

El Hamaoui Y, Yaalaoui S, Chihabeddine K, Boukind E, Moussaoui D (2006) Depression in mothers of burned children. Arch Womens Ment Health 9:117-119

Kornhaber R, Childs C, Cleary M (2018) Experiences of guilt, shame and blame in those affected by burns: a qualitative systematic review. Burns 44:1026-1039

Kraft R et al (2012) Burn size and survival probability in pediatric patients in modern burn care. Lancet 379:1013-1021
Lernevall LST, Moi AL, Cleary M, Kornhaber R, Dreyer P (2020) Support needs of parents of hospitalised children with a burn injury: an integrative review. Burns 46(4):771-781

McGarry S et al (2014) Paediatric burns: from the voice of the child. Burns 40:606-615

McGarry S et al (2015) "This is not just a little accident": a qualitative understanding of paediatric burns from the perspective of parents. Disabil Rehabil 37:41-50

Morley J, Holman N, Murray CD (2017) Dressing changes in a burns unit for children under the age of five: a qualitative study of mothers' experiences. Burns 43:757-765

Pan R et al (2018) School reintegration of pediatric burn survivors: an integrative literature review. Burns 44:494-511

Paulinchen eV (2021) Initiative für brandverletzte Kinder. https:// www.paulinchen.de/. Zugegriffen: 6. Sept. 2021

Phillips C, Rumsey N (2008) Considerations for the provision of psychosocial services for families following paediatric burn injury-a quantitative study. Burns 34:56-62

Ravindran V, Rempel GR, Ogilvie L (2013) Embracing survival: a grounded theory study of parenting children who have sustained burns. Burns 39:589-598

Schaeffer D (Hrsg) (2012) Lehrbuch Patientenberatung, 2. Aufl. Huber, Bern

Schewior-Popp S, Sitzmann F, Ullrich L (Hrsg) (2017) Thiemes Pflege: Das Lehrbuch für Pflegende in Ausbildung, 13. Aufl. Thieme, Stuttgart

Shahsavari H, Matourypour P, Ghiyasvandian S, Golestan Nejad MR (2020) Medical research council framework for development and evaluation of complex interventions: a comprehensive guidance. J Edu Health Promot 9:88

Smith M, Doctor M, Boulter T (2004) Unique considerations in caring for a pediatric burn patient: a developmental approach. Crit Care Nurs Clin North Am 16:99-108

Smith AL, Deborah AM, McBride CJ, McBride-Henry K (2011) A comparison of nurses' and parents' or caregivers' perceptions during pediatric burn dressing changes: an exploratory study. J Burn Care Res 32:185-199

Spronk I, Legemate CM, Polinder S, van Baar ME (2018) Health-related quality of life in children after burn injuries: a systematic review. J Trauma Acute Care Surg 85:1110-1118

Stoddard FJ et al (2017) Posttraumatic stress disorder diagnosis in young children with burns. J Burn Care Res 38:e343-e351

Suurmond J, Dokter J, Van Loey N, Essink-Bot ML (2012) Issues to address in burn care for ethnic minority children: a qualitative study of the experiences of health care staff. Burns 38:730-737

Suurmond J, Bakker A, Van Loey NE (2020) Psychological distress in ethnic minority parents of preschool children with burns. Burns 46:407-415

Willebrand M, Sveen J (2016) Perceived support in parents of children with burns. Gen Hosp Psychiatry 38:105-108

Willebrand M, Sjöberg F, Huss F, Sveen J (2018) Parents' perceived quality of pediatric burn care. J Crit Care 43:256-259

Zegelin A, Ouatedem Tolsdorf M, Umlauf-Beck S (Hrsg) (2009) Patientenedukation: Kurzlehrbuch für Pflege- und Gesundheitsberufe, 2. Aufl. Huber, Bern

Hinweis des Verlags Der Verlag bleibt in Hinblick auf geografische Zuordnungen und Gebietsbezeichnungen in veröffentlichten Karten und Institutsadressen neutral. 